\title{
ON THE UNIQUENESS OF THE (2,2)-DIMENSIONAL SUPERTORUS ASSOCIATED TO A NONTRIVIAL REPRESENTATION OF ITS UNDERLYING 2-TORUS, AND HAVING NONTRIVIAL ODD BRACKETS
}

\author{
R. PENICHE, O. A. SÁNCHEZ-VALENZUELA, and F. THOMPSON
}

Received 12 May 2003

\begin{abstract}
It is proved that up to isomorphism there is only one $(2,2)$-dimensional supertorus associated to a nontrivial representation of its underlying 2-torus, and that it has nontrivial odd brackets. This supertorus is obtained by finding out first a canonical form for its Lie superalgebra, and then using Lie's technique to represent it faithfully as supervector fields on a supermanifold. Those supervector fields can be integrated, and through their various integral flows the composition law for the supergroup is straightforwardly deduced. It turns out that this supertorus is precisely the supergroup described by Guhr (1993) following a formal analogy with the classical unitary group $U(2)$ but with no further intrinsic characterization.
\end{abstract}

2000 Mathematics Subject Classification: 17B70, 58A50, 58C50, 81R05.

1. Introduction and motivation. A physical motivation for the problem we address in this paper is that spacetime is sometimes identified-locally, at least-with the Lie algebra of the unitary group $U(2)$. In fact, $\mathfrak{u}_{2}=\operatorname{Lie}(U(2))$ has the convenient basis defined by Pauli matrices in terms of which an arbitrary $x \in \mathfrak{u}_{2}$ can be written as

$$
x=i\left(\begin{array}{cc}
x_{0}+x_{3} & x_{1}-i x_{2} \\
x_{1}+i x_{2} & x_{0}-x_{3}
\end{array}\right) \quad \text { with } \operatorname{det}(x)=x_{1}^{2}+x_{2}^{2}+x_{3}^{2}-x_{0}^{2} .
$$

It was proved in [4] that making only the most natural assumptions on the Lie algebra $\mathfrak{u}_{2}$ to build up a Lie superalgebra out of $\mathfrak{u}_{2}$, one obtains a 3-parameter family of $(4,4)$ dimensional real Lie superalgebras $\mathfrak{u}_{2}(\lambda, \mu, v)$ whose underlying Lie algebra is $\mathfrak{u}_{2}$ itself. It was further proved there that this 3-parameter family of Lie superalgebras gives rise to ten different isomorphism classes labeled by the number of parameters that are different from zero, and keeps the sign of the product $\lambda v$ constant. Now, the point is that there is a $(2,2)$-dimensional maximal toral subsuperalgebra inside each of these ten different $(4,4)$-dimensional unitary Lie superalgebras. Not all of them, however, are different. It was also shown in [4] that there are only seven different isomorphism classes of $(2,2)$-dimensional toral Lie superalgebras. All of them are associated to the trivial (which in the toral case coincides with the adjoint) representation of the underlying torus in its "odd 2-dimensional sector", and exactly one of them has trivial brackets restricted to the odd sector. What we prove in this paper is that if one is interested in a (2,2)-dimensional Lie superalgebra having the real 2-dimensional Lie algebra of the torus as its underlying Lie algebra, giving rise to a nontrivial action of it on the odd 
sector, and having nontrivial odd brackets, then there is, up to isomorphism, only one such case, namely, the (2,2)-dimensional torus studied by Guhr in [1]. By establishing the uniqueness of this nontrivial supertorus, its relevance is now brought to the foreground, and it can be identified easier as it keeps appearing in the literature under different disguises. Furthermore, it sits inside any of the different $(4,4)$-dimensional unitary Lie supergroups build out of the different Lie superalgebras $\mathfrak{u}_{2}(\lambda, \mu, v)$. These facts are now accompanied in this paper by a set of appealing elementary and straighforward proofs, thus shedding some light into the nature of possible additional structures for superspacetime. The sense that one should provide for the "determinant" that yields the Minkowski metric will be given elsewhere.

2. Statement of the problem and description of the results. Let $\mathbb{F}$ stand for either the real or the complex number fields. A real or complex Lie superalgebra is a $\mathbb{Z}_{2}$-graded vector space $\mathfrak{g}=\mathfrak{g}_{0} \oplus \mathfrak{g}_{1}$ equipped with its grading map $|\cdot|:\left(\mathfrak{g}_{0}-\{0\}\right) \cup\left(\mathfrak{g}_{1}-\{0\}\right) \rightarrow \mathbb{Z}_{2}$ and a bilinear map $\mathbb{[} \cdot, \cdot \mathbb{\|}: \mathfrak{g} \times \mathfrak{g} \rightarrow \mathbb{F}$ satisfying the following properties:

(1) $\llbracket \mathfrak{g}_{\mu}, \mathfrak{g}_{v} \rrbracket \subset \mathfrak{g}_{\mu+v}$,

(2) $\llbracket x, y \rrbracket=-(-1)^{|x||y|} \llbracket y, x \rrbracket$, for any elements $x$ and $y$ in the domain of $|\cdot|$,

(3) $(-1)^{|x||z|} \llbracket \llbracket x, y \rrbracket, z \rrbracket+(-1)^{|z||y|} \llbracket \llbracket z, x \rrbracket, y \rrbracket+(-1)^{|y||x|} \llbracket \llbracket y, z \rrbracket, x \rrbracket=0$, for any elements $x, y$, and $z$ in the domain of $|\cdot|$.

It is well known [5] that this is equivalent to the following structures.

(a) A Lie algebra $\mathfrak{g}_{0}$, with Lie bracket $[\cdot, \cdot]=\llbracket \cdot,\left.\cdot \mathbb{}\right|_{\mathfrak{g}_{0} \times \mathfrak{g}_{0}}$.

(b) A representation $\rho: \mathfrak{g}_{0} \rightarrow$ End $\mathfrak{g}_{1}$, so that $\rho(x) u=\llbracket x, u \rrbracket$.

(c) A symmetric bilinear map $\Gamma: \mathfrak{g}_{1} \times \mathfrak{g}_{1} \rightarrow \mathfrak{g}_{0}$, with $\Gamma(u, v)=\llbracket u, v \rrbracket$, satisfying the following super-Jacobi identities:

(J1)

$$
[x, \Gamma(u, v)]=\Gamma(\rho(x) u, v)+\Gamma(u, \rho(x) v)
$$

for any $x \in \mathfrak{g}_{0}$ and any $u$, and $v$ in $\mathfrak{g}_{1}$, and

(J2)

$$
\rho(\Gamma(u, v))(w)+\rho(\Gamma(w, u))(v)+\rho(\Gamma(v, w))(u)=0
$$

for any $u, v$, and $w$ in $\mathfrak{g}_{1}$.

There are two extreme examples where both conditions are trivially satisfied:

(1) $\rho$ is identically zero,

(2) $\Gamma$ is identically zero.

The case $\rho=0$ of superalgebras associated to the trivial representation occurs in the following nontrivial setting (see [4]). Suppose $\mathfrak{g}_{0}=\mathfrak{g}_{1}$ and $\rho=\mathrm{ad}$, the adjoint representation. If, furthermore, $\mathfrak{g}_{0}$ is the abelian Lie algebra, ad $=0$. For example, when $\mathfrak{g}_{0}$ is the 2-dimensional real Lie algebra of the torus, one might pose the problem of classifying the nonisomorphic Lie superalgebras that can be built upon $\mathfrak{g}_{0}$ associated to the adjoint representation. The answer to this problem has been given in [4], and it turns out that there are seven such cases. On the other hand, the case $\Gamma=0$ also appears on the nontrivial superalgebras studied in [2], with applications to geometry and topology. 
It is our purpose to determine, up to isomorphism, all the real Lie superalgebras that can be defined upon the Lie algebra $\mathfrak{g}_{0}$ of the 2-dimensional torus associated to a real 2-dimensional representation space $\mathfrak{g}_{1}$ for which neither $\rho$ nor $\Gamma$ are identically zero; thus, it is assumed that $\rho: \mathfrak{g}_{0} \rightarrow \mathfrak{g l}\left(\mathfrak{g}_{1}\right)$ satisfies $\operatorname{Exp}(t \rho(x)) \in O\left(\mathfrak{g}_{1}\right)$, for all $x \in \mathfrak{g}_{0}$, all $t \in \mathbb{R}$, and $O\left(\mathfrak{g}_{1}\right)=\left\{\mathfrak{g} \in \mathrm{GL}\left(\mathfrak{g}_{1}\right) \mid \forall u, v \in \mathfrak{g}_{1},(g u, g v)=(u, v)\right\}$, where $(\cdot, \cdot)$ stands for the ordinary scalar product in the 2-dimensional real space $\mathfrak{g}_{1}$. It is proved that, up to isomorphism, there is only one such Lie superalgebra. We also find its associated Lie supergroup and show that it is isomorphic to the supertorus studied in [1].

Convention. Throughout this work, $\mathfrak{g}_{0}=\operatorname{Lie}\left(S^{1} \times S^{1}\right)$, in the sense that the only Lie group we are interested in recovering from $\mathfrak{g}_{0}$ is $S^{1} \times S^{1}$; thereby explaining the assumption on $\rho$.

We proceed as follows. We first use the super-Jacobi identity (J1) to show that if $\rho$ is a nontrivial 2-dimensional representation of the Lie algebra $\mathfrak{g}_{0}$, then $\rho$ determines $\Gamma$ up to scalars (see Proposition 3.1). We then use the super-Jacobi identity (J2) to show in Proposition 3.3 that a certain relationship must hold true amongst the scalars that Proposition 3.1 left free. At this point we realize that any Lie superalgebra on the torus associated to a nontrivial 2-dimensional representation with a nontrivial symmetric $\mathfrak{g}_{0^{-}}$ valued pairing $\Gamma$ can be specified by 5 real parameters with which $\rho$ and $\Gamma$ are built up. We then discuss the natural action of the group $\operatorname{Aut}\left(\mathfrak{g}_{0}\right) \times \mathrm{GL}\left(\mathfrak{g}_{1}\right)$ on the set of pairs $(\rho, \Gamma)$ and show that it acts transitively on the set of such nontrivial pairs, thus showing that there is only one $(2,2)$-dimensional Lie superalgebra based on the torus, for which neither $\rho$, nor $\Gamma$ are zero. A convenient canonical form for such a Lie superalgebra is given as follows in Theorem 4.3: there is a choice of bases $\left\{e_{1}, e_{2}\right\}$ of $\mathfrak{g}_{0}$, and $\left\{f_{1}, f_{2}\right\}$ of $\mathfrak{g}_{1}$ with respect to which

$$
\begin{gathered}
\llbracket e_{1}, f_{1} \rrbracket=f_{2}, \quad \llbracket e_{1}, f_{2} \rrbracket=-f_{1}, \quad \llbracket e_{2}, f_{1} \rrbracket=0, \quad \llbracket e_{2}, f_{2} \rrbracket=0, \\
\llbracket f_{1}, f_{1} \rrbracket=e_{2}, \quad \llbracket f_{1}, f_{2} \rrbracket=0, \quad \llbracket f_{2}, f_{2} \rrbracket=e_{2} .
\end{gathered}
$$

To describe its associated Lie supergroup, let $\left(e^{i s}, e^{i t}\right)$ be the standard coordinates on the 2-torus (that is, $s, t \in \mathbb{R}$ ), and let $\sigma$ and $\tau$ be the odd coordinates. The product $\left(e^{i s}, e^{i t} ; \sigma, \tau\right) *\left(e^{i s^{\prime}}, e^{i t^{\prime}} ; \sigma^{\prime}, \tau^{\prime}\right)$ can be best described in terms of the complex-valued odd variable $\phi=\sigma-i \tau$, and by reading off the result at the end in terms of its real and imaginary parts. Thus

$$
\left(e^{i s}, e^{i t} ; \phi\right) *\left(e^{i s^{\prime}}, e^{i t^{\prime}} ; \phi^{\prime}\right)=\left(e^{i\left(s+s^{\prime}\right)}, e^{i\left(t+t^{\prime}-(1 / 2) \operatorname{Re}\left(e^{-i s^{\prime}} \bar{\phi}^{\prime} \phi\right)\right)} ; \phi^{\prime}+e^{-i s^{\prime}} \phi\right) .
$$

Note the nontrivial effect of the odd coordinates in the "body" of the underlying smooth torus through the term $(-1 / 2) \operatorname{Re}\left(e^{-i s^{\prime}} \bar{\phi}^{\prime} \phi\right)$.

\section{3. $\rho$ determines $\Gamma$ through the Jacobi identities}

Proposition 3.1. Let $\left\{e_{1}, e_{2}\right\}$ be a basis of $\mathfrak{g}_{0}$, and let $\left\{f_{1}, f_{2}\right\}$ be a basis of $\mathfrak{g}_{1}$. Assume $\rho\left(e_{1}\right)=\left(\begin{array}{ll}a & b \\ c & d\end{array}\right)$ with respect to $\left\{f_{1}, f_{2}\right\}$. If (J1) holds true, and $\rho\left(e_{1}\right) \neq 0$ exponentiates to 
a rotation, then $d=-a$ and there are real constants $x_{1}$, and $x_{2}$, such that

$$
\Gamma^{i}=x_{i} J \rho\left(e_{1}\right), \quad i=1,2,
$$

where $J=\left(\begin{array}{cc}0 & -1 \\ 1 & 0\end{array}\right)$. Similarly, write, $\rho\left(e_{2}\right)=\left(\begin{array}{cc}\alpha & \beta \\ \gamma & \delta\end{array}\right)$ with respect to $\left\{f_{1}, f_{2}\right\}$. If $(J 1)$ holds true, and $\rho\left(e_{2}\right) \neq 0$ exponentiates to a rotation, then $\delta=-\alpha$, and there are real constants $y_{1}$, and $y_{2}$, such that

$$
\Gamma^{i}=y_{i} J \rho\left(e_{2}\right), \quad i=1,2 .
$$

In particular, if $\rho\left(e_{1}\right) \neq 0$, and $\rho\left(e_{2}\right) \neq 0$, then, $x_{i} \rho\left(e_{1}\right)=y_{i} \rho\left(e_{2}\right)$ for $i=1,2$.

Proof. We will only work in detail the statement for $\rho\left(e_{1}\right)$. The one for $\rho\left(e_{2}\right)$ is analogous. Note that $\rho\left(e_{1}\right)$ exponentiates to a rotation if and only if its characteristic polynomial has complex conjugate roots, which is true if and only if $(a+d)^{2}-4(a d-$ $b c)<0$; that is, if and only if $(a-d)^{2}+4 b c<0$. It then follows that $a d-b c \neq 0$ and $b c \neq 0$.

Now, write $\Gamma\left(f_{i}, f_{j}\right)=\Gamma_{i j}^{1} e_{1}+\Gamma_{i j}^{2} e_{2}$, and see that putting $x=e_{1}$ in (J1), one obtains

$$
\left(\begin{array}{ll}
a & c \\
b & d
\end{array}\right)\left(\begin{array}{l}
\Gamma_{11}^{i} \\
\Gamma_{12}^{i}
\end{array}\right)=-\left(\begin{array}{c}
0 \\
a \Gamma_{12}^{i}+c \Gamma_{22}^{i}
\end{array}\right), \quad b \Gamma_{12}^{i}+d \Gamma_{22}^{i}=0, \quad i=1,2
$$

Since the matrix is invertible, and $b \neq 0$, we may write $\Gamma_{12}^{i}=-(d / b) \Gamma_{22}^{i}$, and make the corresponding substitution in the matrix equation to get $\Gamma_{11}^{i}=-(c / b) \Gamma_{22}^{i}$, and $(a+$ d) $\Gamma_{22}^{i}=0$.

It is now clear that either $\Gamma=0$, or there exists some $i \in\{1,2\}$ for which $\Gamma_{22}^{i} \neq 0$, in which case $a+d=0$, and setting $x_{i}=\Gamma_{22}^{i} / b$ we can write $\Gamma^{i}$ as in the statement. The last assertion in the statement is clear.

REMARK 3.2. If we were not interested in representations of the torus we would not have had to require that $\rho\left(e_{i}\right)$ had a characteristic polynomial with complex conjugate roots. If we were only interested in having a nontrivial representation of the 2-dimensional abelian Lie algebra $\mathfrak{g}_{0} \simeq \mathbb{R}^{2}$ in the 2-dimensional odd sector, then (J1) yields nontrivial $\Gamma$ 's if and only if $a \gamma=c \alpha$ and $b \delta=d \beta$.

Table 3.1 summarizes what we have just found. Note from the proof of Proposition 3.1 that not all the parameters are independent, since $x_{1} y_{2}=x_{2} y_{1}$.

Proposition 3.3. Under the hypotheses of Proposition 3.1, (J2) yields

$$
x_{1}=-\lambda x_{2}, \quad y_{2}=-v y_{1}
$$

In particular, if $\lambda=0$ (i.e., $\rho_{2}=0$ ), then $\Gamma^{1}=0$, whereas if $\nu=0$ (i.e., $\rho_{1}=0$ ), then $\Gamma^{2}=0$. 
TABLE 3.1

\begin{tabular}{ccccc}
\hline$\rho\left(e_{1}\right)$ & $\rho\left(e_{2}\right)$ & $\Gamma^{1}$ & $\Gamma^{2}$ & $x_{1} y_{2}=x_{2} y_{1}$ \\
\hline$\left(\begin{array}{cc}a & b \\
c & -a\end{array}\right)$ & $\lambda \rho\left(e_{1}\right)$ & $x_{1} J \rho\left(e_{1}\right)$ & $x_{2} J \rho\left(e_{1}\right)$ & $\lambda=0$ or $\exists k, \lambda=x_{k} / y_{k}$ \\
$\nu \rho\left(e_{2}\right)$ & $\left(\begin{array}{cc}\alpha & \beta \\
\gamma & -\alpha\end{array}\right)$ & $y_{1} J \rho\left(e_{2}\right)$ & $y_{2} J \rho\left(e_{2}\right)$ & $v=0$ or $\exists k, v=y_{k} / x_{k}$ \\
\hline
\end{tabular}

Proof. Note that (J2) implies

$$
\left(\Gamma_{11}^{1}+\lambda \Gamma_{11}^{2}\right)\left(\begin{array}{l}
a \\
c
\end{array}\right)=\left(\begin{array}{l}
0 \\
0
\end{array}\right), \quad\left(v \Gamma_{11}^{1}+\Gamma_{11}^{2}\right)\left(\begin{array}{l}
\alpha \\
\gamma
\end{array}\right)=\left(\begin{array}{l}
0 \\
0
\end{array}\right) .
$$

Since $b c \neq 0$ when $\rho\left(e_{1}\right) \neq 0$, we have $\Gamma_{11}^{1}+\lambda \Gamma_{11}^{2}=0$. Similarly, $\beta \gamma \neq 0$, when $\rho\left(e_{2}\right) \neq 0$, in which case $v \Gamma_{11}^{1}+\Gamma_{11}^{2}=0$.

OBSERVATION 3.4. So far, we have obtained a parametric description of any $(2,2)$ dimensional Lie superalgebra having $\mathfrak{g}_{0}=\operatorname{Lie}\left(S^{1} \times S^{1}\right)$, compatible $\rho \neq 0$, and $\Gamma \neq 0$. We have proved that any such superalgebra depends on 5 real parameters. We now want to see whether given two such sets of five parameters, the Lie superalgebras they give rise to, are isomorphic or not. This is done in Section 4 below.

ObSERVATION 3.5. Write $\Gamma(u, v)=\Gamma^{1}(u, v) e_{1}+\Gamma^{2}(u, v) e_{2}$, with $\Gamma^{i}(i=1,2)$ a real symmetric bilinear form on $\mathfrak{g}_{1}$. Write $O_{\Gamma^{i}}\left(\mathfrak{g}_{1}\right)$ for the group $\left\{g \in G L\left(\mathfrak{g}_{1}\right) \mid \Gamma^{i}(\mathfrak{g u}, \mathfrak{g} v)=\right.$ $\left.\Gamma^{i}(u, v)\right\}$. If $\Gamma^{i}$ is nondegenerate then it defines a scalar product in $\mathfrak{g}_{1}$, and $O_{\Gamma^{i}}\left(\mathfrak{g}_{1}\right)$ is actually an orthogonal group. It follows from (J1) that for all $x \in \mathfrak{g}_{0}, \rho(x) \in \operatorname{Lie}\left(O_{\Gamma^{i}}\right)$. The proof of Proposition 3.1 shows (see Table 3.1 above) that $\Gamma^{1}$ and $\Gamma^{2}$ are scalar multiples of a single bilinear form, say $\Gamma^{0}$, whose matrix in the basis $\left\{f_{1}, f_{2}\right\}$ is either $J \rho\left(e_{1}\right)$ or $J \rho\left(e_{2}\right)$. Here an alternative argument is presented. If $\operatorname{Exp}(t \rho(x)) \in O\left(\mathfrak{g}_{1}\right)=\{g \in$ $\left.\operatorname{GL}\left(\mathfrak{g}_{1}\right) \mid \forall u, v \in \mathfrak{g}_{1},(g u, g v)=(u, v)\right\}$, where $(\cdot, \cdot)$ stands for the ordinary scalar product in the 2-dimensional space $\mathfrak{g}_{1}$, then $\Gamma^{0}=c(\cdot, \cdot)$ for some nonzero real scalar $c$. Whence, $\Gamma^{i}=c_{i}(\cdot, \cdot)(i=1,2)$. In particular, the basis $\left\{f_{1}, f_{2}\right\}$ of $\mathfrak{g}_{1}$ we started with in Proposition 3.1 could have been orthogonal. This would have made $\Gamma_{i j}^{k}=0$ for $i \neq j$, and would have eliminated the off-diagonal parameters $\Gamma_{12}^{k}$. Since we were going to classify all Lie superalgebras with the stated properties, there was no point for choosing the orthonormal basis at this point, and we preferred to let that follow at the end of the classification argument (the basis $\left\{f_{1}, f_{2}\right\}$ in Theorem 4.3 below turns out to be orthogonal). Nevertheless, the reader might have preferred to introduce an orthogonal basis at this earlier stage, and then use $S \in O\left(\mathfrak{g}_{1}\right)$ instead of $S \in \mathrm{GL}\left(\mathfrak{g}_{1}\right)$ in Section 4 below. We are indebted to one of the referees for pointing this out to us.

4. Classification up to isomorphism. Two Lie superalgebras $\mathfrak{g}$ and $\mathfrak{g}^{\prime}$ are isomorphic if and only if there exists a pair $(T, S)$ where $T: \mathfrak{g}_{0} \rightarrow \mathfrak{g}_{0}^{\prime}$ is a Lie algebra isomorphism, and $S: \mathfrak{g}_{1} \rightarrow \mathfrak{g}_{1}^{\prime}$ is a linear isomorphism such that

$$
\llbracket(T \oplus S)(\cdot),(T \oplus S)(\cdot) \rrbracket^{\prime}=(T \oplus S)(\llbracket \cdot, \cdot \rrbracket) .
$$


Since, a Lie superalgebra $\mathfrak{g}=\mathfrak{g}_{0} \oplus \mathfrak{g}_{1}$ can be described by the data $\left(\mathfrak{g}_{0}, \rho, \Gamma\right)$ satisfying $(\mathrm{J} 1)$ and (J2), the isomorphism conditions can be read in the following form:

$$
[\cdot, \cdot]^{\prime}=T\left[T^{-1}(\cdot), T^{-1}(\cdot)\right], \quad \rho^{\prime}=S \circ \rho\left(T^{-1}(\cdot)\right) \circ S^{-1}, \quad \Gamma^{\prime}=T\left(\Gamma\left(S^{-1}(\cdot), S^{-1}(\cdot)\right)\right) .
$$

Now, for $\mathfrak{g}_{0}=\mathfrak{g}_{0}^{\prime}$, the first condition simply says that $T \in \operatorname{Aut}\left(\mathfrak{g}_{0}\right)$. We now want to act on the pairs $(\rho, \Gamma)$ with $(T, S) \in \operatorname{Aut}\left(\mathfrak{g}_{0}\right) \times \operatorname{GL}\left(\mathfrak{g}_{1}\right)$ in order to determine the isomorphism classes defined by them.

LEMMA 4.1. Let $\rho$ stand for either $\rho\left(e_{1}\right)$ or $\rho\left(e_{2}\right)$ as described above, and let $\Gamma=z J \rho$ as in Proposition 3.1. Let $S \in \mathrm{GL}\left(\mathfrak{g}_{1}\right)$ be such that $S \rho S^{-1}=\mu \mathrm{J}$, then

$$
\left(S^{-1}\right)^{t} \Gamma S^{-1}=\frac{-z \mu}{\operatorname{det} S} I
$$

where $I=\left(\begin{array}{ll}1 & 0 \\ 0 & 1\end{array}\right)$.

Proof. We use the fact that for any $2 \times 2$-matrix $P$, we have $P^{t} J P=\operatorname{det} P J$. Thus if $\Gamma=z J \rho$, we get

$$
\left(S^{-1}\right)^{t} \Gamma S^{-1}=z\left(S^{-1}\right)^{t} J S^{-1} S \rho S^{-1}=z \mu \operatorname{det}\left(S^{-1}\right) J^{2} .
$$

LEMMA 4.2. Let $\rho^{\prime}=S \circ \rho\left(T^{-1}(\cdot)\right) \circ S^{-1}$ and $\Gamma^{\prime}=T\left(\Gamma\left(S^{-1}(\cdot), S^{-1}(\cdot)\right)\right)$.

(1) Suppose $\rho\left(e_{2}\right)=\lambda \rho\left(e_{1}\right)$, so that $\Gamma^{1}=-\lambda \Gamma^{2}$, and $\Gamma^{2}=x_{2} J \rho\left(e_{1}\right)$. Then,

$$
\begin{array}{ll}
\rho^{\prime}\left(e_{1}\right)=\frac{T_{22}-\lambda T_{21}}{\operatorname{det} T} S \rho\left(e_{1}\right) S^{-1}, & \Gamma^{\prime 1}=\left(-T_{11} \lambda+T_{12}\right)\left(S^{-1}\right)^{t} \Gamma^{2} S^{-1}, \\
\rho^{\prime}\left(e_{2}\right)=\frac{-T_{12}+\lambda T_{11}}{\operatorname{det} T} S \rho\left(e_{1}\right) S^{-1}, & \Gamma^{\prime 2}=\left(-T_{21} \lambda+T_{22}\right)\left(S^{-1}\right)^{t} \Gamma^{2} S^{-1} .
\end{array}
$$

(2) Suppose $\rho\left(e_{1}\right)=v \rho\left(e_{2}\right)$, so that $\Gamma^{2}=-v \Gamma^{1}$, and $\Gamma^{1}=y_{1} J \rho\left(e_{2}\right)$. Then,

$$
\begin{array}{ll}
\rho^{\prime}\left(e_{1}\right)=\frac{v T_{22}-T_{21}}{\operatorname{det} T} S \rho\left(e_{2}\right) S^{-1}, & \Gamma^{\prime 1}=\left(T_{11}-v T_{12}\right)\left(S^{-1}\right)^{t} \Gamma^{1} S^{-1}, \\
\rho^{\prime}\left(e_{2}\right)=\frac{-v T_{12}+T_{11}}{\operatorname{det} T} S \rho\left(e_{2}\right) S^{-1}, & \Gamma^{\prime 2}=\left(T_{21}-v T_{22}\right)\left(S^{-1}\right)^{t} \Gamma^{1} S^{-1} .
\end{array}
$$

The proof of Lemma 4.2 is a straightforward and immediate computation.

Our main result is now an immediate consequence of the two previous lemmas.

THEOREM 4.3. There is, up to isomorphism, only one $(2,2)$-dimensional Lie superalgebra on the torus with nontrivial $\rho$ and nontrivial $\Gamma$. In fact, there is a basis $\left\{e_{1}, e_{2}\right\}$ of $\mathfrak{g}_{0}$ and $\left\{f_{1}, f_{2}\right\}$ of $\mathfrak{g}_{1}$ such that (2.1) holds.

Proof. In either of the two statements of Lemma 4.2, we can choose $S$ so that $S \rho\left(e_{i}\right) S^{-1}=\mu_{i} J$, and choose $T$ and $\operatorname{det} S$ so that $\rho^{\prime}\left(e_{1}\right)=J, \rho^{\prime}\left(e_{2}\right)=0, \Gamma^{\prime 1}=0$, and $\Gamma^{\prime 2}=I$. 
5. The Lie supergroup associated to the nontrivial torus. It is a remarkable fact that this Lie superalgebra sits inside of almost any of the unitary Lie superalgebras $\mathfrak{u}_{2}(\lambda, \mu, v)$ described in [4]. In fact, the only condition to provide an embedding is $v \neq 0$. To prove this fact, look first at the real Lie algebra $\mathfrak{u}_{2}$ of the unitary group $U_{2}$. A convenient basis for it is $\{i I, i H, E-F, i(E+F)\}$ where

$$
I=\left(\begin{array}{ll}
1 & 0 \\
0 & 1
\end{array}\right), \quad H=\left(\begin{array}{cc}
1 & 0 \\
0 & -1
\end{array}\right), \quad E=\left(\begin{array}{ll}
0 & 1 \\
0 & 0
\end{array}\right), \quad F=\left(\begin{array}{ll}
0 & 0 \\
1 & 0
\end{array}\right) .
$$

Now, look at the set $\{\pi(i I), \pi(i H), \pi(E-F), \pi(i(E+F))\}$ regarded as a basis for an odd copy of $\mathfrak{u}_{2}$ as in [4]. Let $\mathfrak{u}_{2}(\lambda, \mu, v)$ be the Lie superalgebra structure on $\mathfrak{u}_{2} \oplus \mathfrak{u}_{2}$ associated to the adjoint representation and the real parameters $(\lambda, \mu, v)$ as in [4]. It is then clear that the map

$$
\begin{array}{ll}
e_{1} \longmapsto \frac{i}{2} H, & f_{1} \longmapsto \sqrt{-i} \pi(E-F), \\
e_{2} \longmapsto 2 i I, & f_{2} \longmapsto i \sqrt{-i} \pi(E+F),
\end{array}
$$

is a Lie superalgebra monomorphism.

Moreover, by looking at the representation of the basis vectors $\{2 i I,(i / 2) H ; \sqrt{-i} \pi(E-$ $F), i \sqrt{-i} \pi(E+F)\}$ in terms of supervector fields as in [4], it is a straightforward matter to obtain an explicit expression for the composition law of the corresponding supertorus:

$$
\begin{aligned}
& e_{1} \longleftrightarrow \frac{i}{2}\left(z^{1} \frac{\partial}{\partial z^{1}}-z^{2} \frac{\partial}{\partial z^{2}}+\zeta^{1} \frac{\partial}{\partial \zeta^{1}}-\zeta^{2} \frac{\partial}{\partial \zeta^{2}}\right), \\
& e_{2} \longleftrightarrow 2 i\left(z^{1} \frac{\partial}{\partial z^{1}}+z^{2} \frac{\partial}{\partial z^{2}}+\zeta^{1} \frac{\partial}{\partial \zeta^{1}}+\zeta^{2} \frac{\partial}{\partial \zeta^{2}}\right), \\
& f_{1} \longleftrightarrow \sqrt{-i}\left(z^{1} \frac{\partial}{\partial \zeta^{2}}-z^{2} \frac{\partial}{\partial \zeta^{1}}+\zeta^{1} \frac{\partial}{\partial z^{2}}-\zeta^{2} \frac{\partial}{\partial z^{1}}\right), \\
& f_{2} \longleftrightarrow i \sqrt{-i}\left(z^{1} \frac{\partial}{\partial \zeta^{2}}+z^{2} \frac{\partial}{\partial \zeta^{1}}+\zeta^{1} \frac{\partial}{\partial z^{2}}+\zeta^{2} \frac{\partial}{\partial z^{1}}\right) .
\end{aligned}
$$

The integral flows can be easily computed as in [3] to obtain

$$
\begin{aligned}
& \operatorname{Exp}\left(t e_{2}\right)\left\{\begin{array}{l}
z^{1} \longmapsto e^{2 i t} z^{1}, \quad \zeta^{1} \longmapsto e^{2 i t} \zeta^{1}, \\
z^{2} \longmapsto e^{2 i t} z^{2}, \quad \zeta^{2} \longmapsto e^{2 i t} \zeta^{2},
\end{array}\right. \\
& \operatorname{Exp}\left(s e_{1}\right) \begin{cases}z^{1} \longmapsto e^{i(s / 2)} z^{1}, & \zeta^{1} \longmapsto e^{i(s / 2)} \zeta^{1}, \\
z^{2} \longmapsto e^{-i(s / 2)} z^{2}, & \zeta^{2} \longmapsto e^{-i(s / 2)} \zeta^{2},\end{cases} \\
& \operatorname{Exp}\left(\tau f_{2}\right) \begin{cases}z^{1} \longmapsto z^{1}+i \sqrt{-i} \tau \zeta^{2}, & \zeta^{1} \longmapsto \zeta^{1}+i \sqrt{-i} \tau z^{2}, \\
z^{2} \longmapsto z^{2}+i \sqrt{-i} \tau \zeta^{1}, & \zeta^{2} \longmapsto \zeta^{2}+i \sqrt{-i} \tau z^{1},\end{cases} \\
& \operatorname{Exp}\left(\sigma f_{1}\right) \begin{cases}z^{1} \longmapsto z^{1}-\sqrt{-i} \sigma \zeta^{2}, & \zeta^{1} \longmapsto \zeta^{1}-\sqrt{-i} \sigma z^{2}, \\
z^{2} \longmapsto z^{2}+\sqrt{-i} \sigma \zeta^{1}, & \zeta^{2} \longmapsto \zeta^{2}+\sqrt{-i} \sigma z^{1}\end{cases}
\end{aligned}
$$


We introduce the complex-valued odd variable $\phi=\sigma-i \tau$, in terms of which the (local) superdiffeomorphism $\operatorname{Exp}\left(\sigma f_{1}\right) \circ \operatorname{Exp}\left(\tau f_{2}\right) \circ \operatorname{Exp}\left(s e_{1}\right) \circ \operatorname{Exp}\left(t e_{2}\right)$ is given by

$$
\begin{aligned}
& z^{1} \longmapsto e^{2 i t+(i / 2) s}\left(z^{1}\left(1+\frac{i}{2} \phi \bar{\phi}\right)+\sqrt{-i} \zeta^{2} \phi\right), \\
& \zeta^{1} \longmapsto e^{2 i t+(i / 2) s}\left(\zeta^{1}\left(1+\frac{i}{2} \phi \bar{\phi}\right)-\sqrt{-i} z^{2} \phi\right), \\
& z^{2} \longmapsto e^{2 i t-(i / 2) s}\left(z^{2}\left(1-\frac{i}{2} \phi \bar{\phi}\right)-\sqrt{-i} \zeta^{1} \bar{\phi}\right), \\
& \zeta^{2} \longmapsto e^{2 i t-(i / 2) s}\left(\zeta^{2}\left(1-\frac{i}{2} \phi \bar{\phi}\right)+\sqrt{-i} z^{1} \bar{\phi}\right) .
\end{aligned}
$$

We set $\Phi(s, t ; \sigma, \tau)=\operatorname{Exp}\left(\sigma f_{1}\right) \circ \operatorname{Exp}\left(\tau f_{2}\right) \circ \operatorname{Exp}\left(s e_{1}\right) \circ \operatorname{Exp}\left(t e_{2}\right)$, and find from $\Phi\left(s^{\prime \prime}, t^{\prime \prime}\right.$; $\left.\sigma^{\prime \prime}, \tau^{\prime \prime}\right)=\Phi\left(s^{\prime}, t^{\prime} ; \sigma^{\prime}, \tau^{\prime}\right) \circ \Phi(s, t ; \sigma, \tau)$, the following set of equalities:

$$
\begin{aligned}
& e^{2 i\left(t+t^{\prime}\right)+(i / 2)\left(s+s^{\prime}\right)}\left(\left(1+\frac{i}{2} \phi^{\prime} \bar{\phi}^{\prime}\right)\left(1+\frac{i}{2} \phi \bar{\phi}\right)-i e^{-i s^{\prime}} \bar{\phi}^{\prime} \phi\right) \\
& =e^{2 i t^{\prime \prime}+(i / 2) s^{\prime \prime}}\left(1+\frac{i}{2} \phi^{\prime \prime} \overline{\phi^{\prime \prime}}\right), \\
& e^{2 i\left(t+t^{\prime}\right)+(i / 2)\left(s+s^{\prime}\right)}\left(\phi^{\prime}\left(1+\frac{i}{2} \phi \bar{\phi}\right)+e^{-i s^{\prime}}\left(1-\frac{i}{2} \phi^{\prime} \bar{\phi}^{\prime}\right) \phi\right) \\
& =e^{2 i t^{\prime \prime}+(i / 2) s^{\prime \prime}} \phi^{\prime \prime}, \\
& e^{2 i\left(t+t^{\prime}\right)-(i / 2)\left(s+s^{\prime}\right)}\left(\left(1-\frac{i}{2} \phi^{\prime} \overline{\phi^{\prime}}\right)\left(1-\frac{i}{2} \phi \bar{\phi}\right)-i e^{i s^{\prime}} \phi^{\prime} \bar{\phi}\right) \\
& =e^{2 i t^{\prime \prime}-(i / 2) s^{\prime \prime}}\left(1-\frac{i}{2} \phi^{\prime \prime} \overline{\phi^{\prime \prime}}\right), \\
& e^{2 i\left(t+t^{\prime}\right)-(i / 2)\left(s+s^{\prime}\right)}\left(\bar{\phi}^{\prime}\left(1-\frac{i}{2} \phi \bar{\phi}\right)+e^{i s^{\prime}}\left(1+\frac{i}{2} \phi^{\prime} \bar{\phi}^{\prime}\right) \bar{\phi}\right) \\
& =e^{2 i t^{\prime \prime}-(i / 2) s^{\prime \prime}} \overline{\phi^{\prime \prime}} .
\end{aligned}
$$

The first and third equations can be multiplied side by side in order to solve for $t^{\prime \prime}$. One may also multiply the first with the complex conjugate of the third to solve for $s^{\prime \prime}$. Using the results for $t^{\prime \prime}$ and $s^{\prime \prime}$ in the second equation, one finally solves for $\phi^{\prime \prime}$. The result is the composition law for the supertorus:

$$
\begin{aligned}
t^{\prime \prime} & =t+t^{\prime}-\frac{1}{4}\left(e^{-i s^{\prime}} \bar{\phi}^{\prime} \phi+e^{i s^{\prime}} \phi^{\prime} \bar{\phi}\right), \\
s^{\prime \prime} & =s+s^{\prime}, \\
\phi^{\prime \prime} & =\phi^{\prime}+e^{-i s^{\prime}} \phi .
\end{aligned}
$$

Note that an embedding of this nontrivial $(2,2)$-supertorus can be given into the supergroup (see [4]) $U_{2}(2,2,1)$ by means of the map

$$
\left(e^{i s}, e^{i t} ; \phi\right) \longmapsto\left(\begin{array}{cc}
e^{2 i t} & 0 \\
0 & e^{2 i t}
\end{array}\right)\left(\begin{array}{cc}
e^{i s / 2} & 0 \\
0 & e^{-i s / 2}
\end{array}\right)\left(\begin{array}{cc}
1+\frac{i}{2} \phi \bar{\phi} & \sqrt{-i} \phi \\
\sqrt{-i} \bar{\phi} & 1-\frac{i}{2} \phi \bar{\phi}
\end{array}\right),
$$

which was the way Guhr originally described in [1]. 
ACKNOWLEDGMENTS. The authors would like to acknowledge with thanks the partial support received from the following: the Marsden Fund of the Royal Society of New Zealand, Grant MB-1411-2002, and CONACYT Grant 37558-E. Specially acknowledged are CONACYT's postdoctoral fellowship received by Peniche to spend the 2001-2002 academic year at the Department of Mathematics at MIT, and CONACYT's sabbatical fellowship received by Sánchez-Valenzuela to spend the 2002-2003 academic year at the Department of Mathematics at Harvard University. Last, but not least, we would like to thank Professor Vladimir Pestov for enlightening discussions during the genesis of this work, and the kind hospitality received by the three authors at CIMAT, Victoria University of Wellington, MIT, and Harvard University.

\section{REFERENCES}

[1] T. Guhr, On the graded group U(1/1), J. Math. Phys. 34 (1993), no. 6, 2541-2553.

[2] V. W. Guillemin and S. Sternberg, Supersymmetry and Equivariant de Rham Theory, Mathematics Past and Present, Springer-Verlag, Berlin, 1999.

[3] J. Monterde and O. A. Sánchez-Valenzuela, Existence and uniqueness of solutions to superdifferential equations, J. Geom. Phys. 10 (1993), no. 4, 315-343.

[4] R. Peniche and O. A. Sánchez-Valenzuela, Lie supergroups supported over $\mathrm{GL}_{2}$ and $U_{2}$ associated to the adjoint representation, to appear in J. Geom. Phys.

[5] M. Scheunert, The Theory of Lie Superalgebras, Lecture Notes in Mathematics, vol. 716, Springer, New York, 1979.

R. Peniche: Centro de Investigación en Matemáticas, Apartado Postal 402, 36000 Guanajuato, Mexico

E-mail address: peniche@cimat.mx

Current address: Facultad de Matemáticas, Universidad Autónoma de Yucatán, Mérida, Yucatán, Mexico

E-mail address: pmena@tunku.uady.mx

O. A. Sánchez-Valenzuela: Centro de Investigación en Matemáticas, Apartado Postal 402, 36000 Guanajuato, Mexico

E-mail address: ado1fo@cimat.mx

F. Thompson: Department of Mathematics, Victoria University of Wellington, Wellington 6001, New Zealand

E-mail address: fin1ay@scoop.co.nz 


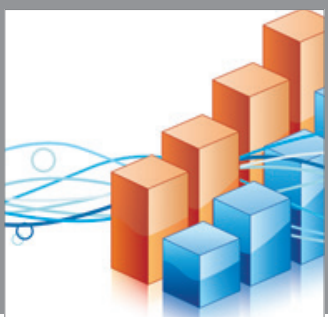

Advances in

Operations Research

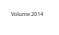

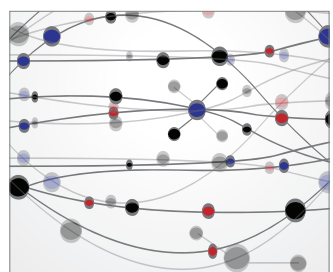

\section{The Scientific} World Journal
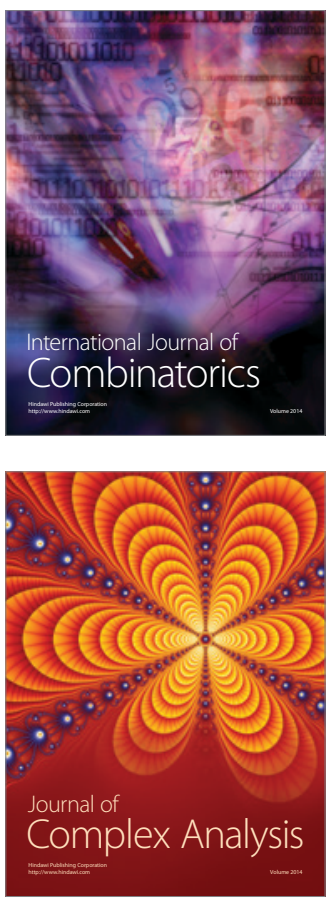

International Journal of

Mathematics and

Mathematical

Sciences
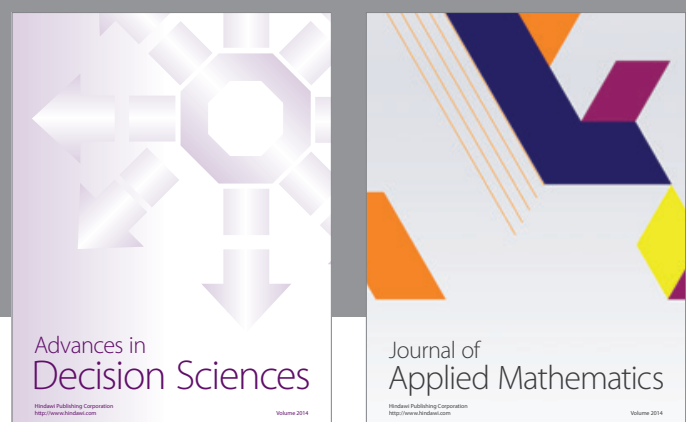

Journal of

Applied Mathematics
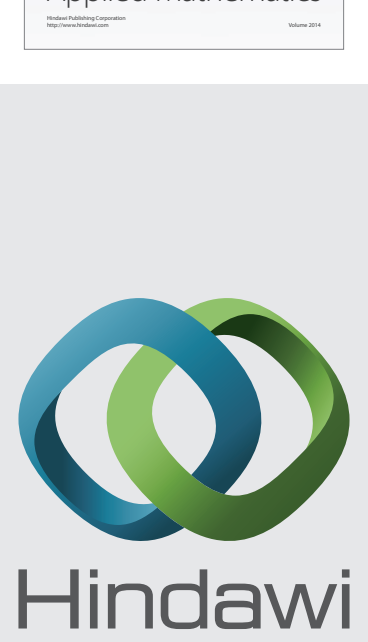

Submit your manuscripts at http://www.hindawi.com
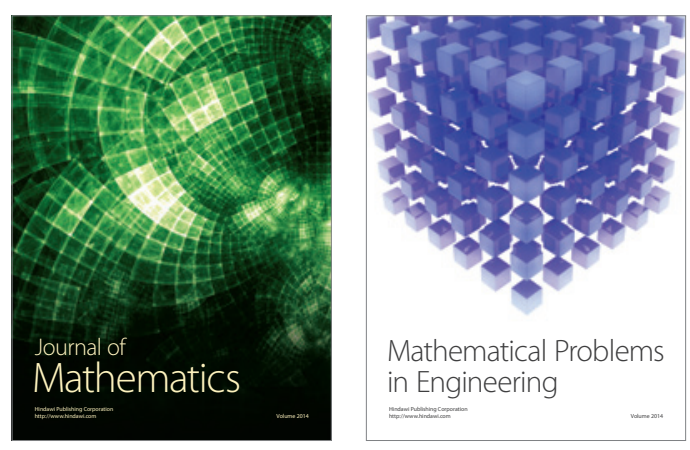

Mathematical Problems in Engineering
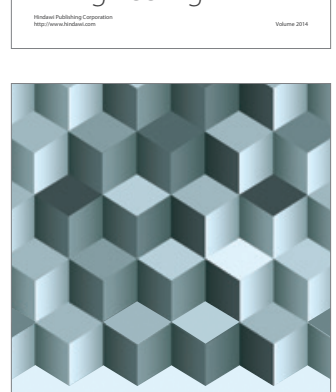

Journal of

Function Spaces
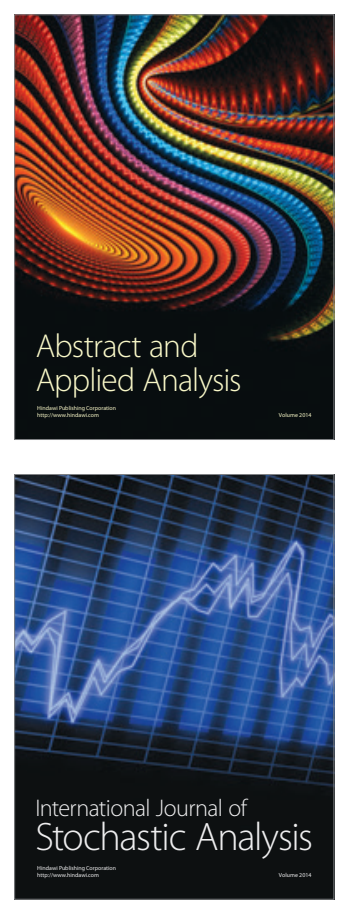

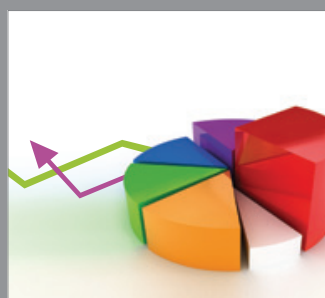

ournal of

Probability and Statistics

Promensencen
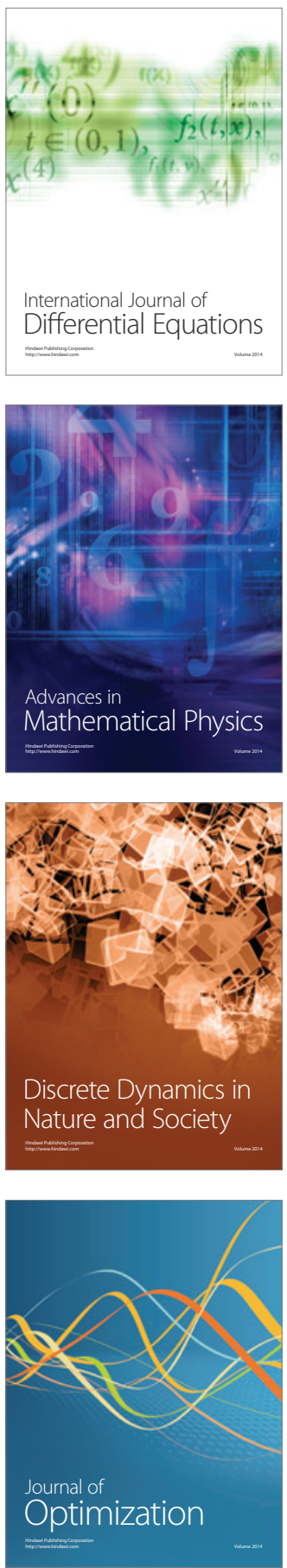